\title{
Un ensayo clínico piloto de la efectividad clínica de dos presentaciones de esomeprazol
}

\section{Clinical effectiveness of two esomeprazole presentations in a pilot trial}

\author{
Carlos Arturo Rojas, ${ }^{1 *}$ Mario Sepúlveda Copete, ${ }^{1}$ Jairo Alberto García Abadía, ${ }^{1}$ Héctor Raúl Echavarría Abad, ${ }^{2}$ Fernando Rosso Suárez, ${ }^{3}$ \\ Andrés Fernando Jiménez, ${ }^{4}$ Andrés Mauricio Castro Llanos. ${ }^{5}$
}

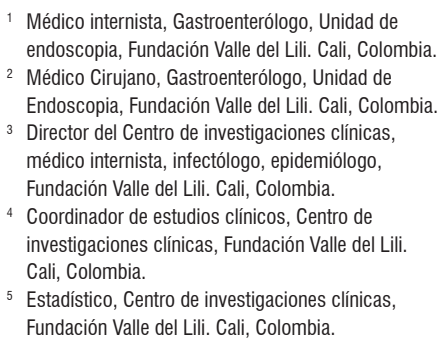

*Correspondencia: crojo16@yahoo.com.

\begin{abstract}
Resumen
Introducción: el presente estudio tuvo como fin investigar la efectividad clínica de dos presentaciones de esomeprazol en pacientes con dispepsia de causa no estudiada. Métodos: se realizó un ensayo clínico piloto de dos presentaciones de esomeprazol de $40 \mathrm{mg}$ recibidos diariamente por 28 días. Se eligieron pacientes con diagnóstico de dispepsia no estudiada que asistieron a consulta de gastroenterología en un hospital de referencia. Se evaluaron a los pacientes inicialmente con endoscopia y biopsia, el seguimiento a 2 y 4 semanas con escalas clínicas de síntomas y calidad de vida con cuestionarios validados en español (SODA y QoL-PEI) y eventos adversos. Además, se midieron los niveles de pH gástrico con pH-metrías en 24 horas al día 14 de tratamiento. Se tomaron niveles séricos del medicamento al momento de la evaluación de la pH-metría. Para las escalas clínicas se aplicó un análisis de varianza (ANOVA) de dos factores con medidas repetidas y al encontrar diferencias significativas en los tiempos se realizó una corrección de Bonferroni. Resultados: se aleatorizó un total de 33 pacientes, 16 y 17 pacientes en cada grupo. No hubo diferencias en el porcentaje de inhibición del pH gástrico al día 14 de tratamiento $(p=0,9795)$. No hubo diferencias en concentraciones de niveles séricos el día $14(p=0,2199)$. No se encontraron diferencias significativas en las escalas de gravedad y calidad de vida en las dos primeras semanas de tratamiento, pero sí en las últimas dos semanas, en las cuales el producto de prueba demostró mayor disminución del dolor $(p=0,0048)$ y superioridad en conformidad $(p=0,01)$ en la subescala SODA. No se presentaron eventos adversos serios y no hubo diferencias estadísticas entre la presentación eventos adversos no serios. Conclusiones: los productos de prueba y el de referencia mostraron efectos similares en variables clínicamente relevantes.
\end{abstract}

Palabras clave

Esomeprazol, SODA, QoL-PEI, inhibidores de la bomba de protones, dispepsia.

\begin{abstract}
Introduction: This pilot studied the clinical effectiveness of two presentations of esomeprazole in patients with dyspepsia with undiagnosed causes. Methods: We conducted a pilot clinical trial of two $40 \mathrm{mg}$ Esomeprazole presentations. Patients with dyspepsia of unknown cause at a gastroenterology clinic in a referral hospital were included. They received one or the other presentation daily for 28 days. Patients were initially evaluated with endoscopy and biopsy and received follow-up examinations at two and four weeks. Adverse events were recorded, and clinical symptom scales and quality of life questionnaires validated in Spanish (SODA and QoL-PEI) were used. In addition, gastric pH levels were measured continuously for 24 hours on day 14 of treatment. Serum levels of the medication administered were also measured on day 14 of treatment. A two-way repeated measures ANOVA was used to compare mean differences between the two groups. When significant differences in times were found, a Bonferroni correction was made. Results: A total of 33 patients were randomized into two groups: 16 patients in one group and 17 in the other. There were no differences in the percentages of gastric $\mathrm{pH}$ inhibition at day 14 of treatment $(p=0.9795)$. There were no differences in serum level concentrations on day $14(p=0.2199)$. No significant differences were found in severity and quality of life scales in the first two weeks of treatment. However, in the last two weeks of treatment the test product showed a larger decrease in pain $(p=0.0048)$ and superiority in compliance $(p=0.01)$ on the SODA subscale. There were no serious adverse events, and there were no statistical differences between the presentations of non-serious adverse events. Conclusions: The Test product and the Reference product showed similar effects on clinically relevant variables.
\end{abstract}

Keywords

Esomeprazole, SODA; QoL-PEI, proton pump inhibitors, dispepsia. 


\section{INTRODUCCIÓN}

La dispepsia se define como una sensación de dolor o malestar abdominal crónico y recurrente localizado en la parte central del abdomen superior (1). Según los criterios del consenso ROMA IV se clasifica en dos tipos: el primero, de causa orgánica definida; y el segundo, sin una causa específica; y se denomina dispepsia funcional (DF). La DF se considera multifactorial y puede deberse a alteraciones fisiológicas, inmunológicas, de hipersensibilidad y de la interacción cerebro-intestino; además, está asociada con hábitos diarios relacionados con estilos de alimentación y vida, e incluye la infección por Helicobacter pylori, de gran importancia en nuestro medio debido a su alta prevalencia, aproximadamente del $60 \%(2-4)$.

Se considera que alrededor del $15 \%$ al $40 \%$ de la población mundial cursa con síntomas dispépticos de los cuales el $70 \%$ son idiopáticos. La incidencia anual de la dispepsia es aproximadamente del $1 \%$ y se calcula que el $50 \%$ de las personas consultará en algún momento de su vida por estos síntomas (1-3).

El impacto negativo que tiene la dispepsia en la calidad de vida ha incentivado el desarrollo de escalas de valoración que permitan medir la gravedad, discapacidad y alteración en los procesos de la vida diaria. Para la comunidad latina se cuenta con dos instrumentos validados al español: la escala SODA (Severity of Dyspepsia Assessment), que valora la intensidad del dolor, síntomas asociados y nivel de conformidad; y la escala QoL-PEI (cuestionario de calidad de vida asociado a problemas intestinales), que determina el impacto que tiene esta enfermedad en la vida diaria (4-11).

Parte del manejo estándar en la dispepsia se ha enfocado en el control del ácido gástrico, y uno de los principales grupos de medicamentos con este fin son los inhibidores de la bomba de protones (IBP), que se han utilizado ampliamente en DF y orgánica (1, 5-8). El esomeprazol es parte de este grupo, está indicado en el alivio de síntomas gastrointestinales, cicatrización de lesiones y mantenimiento de la cicatrización (5). El esomeprazol mejora los síntomas dispépticos mediante varios mecanismos: primero, los pacientes con dispepsia presentan hipersensibilidad duodenal al ácido; y segundo, los pacientes con dispepsia presentan inflamación de bajo grado que es empeorada por la secreción ácida. En las situaciones anteriores, la inhibición de la secreción gástrica mediante esomeprazol modifica el efecto de las alteraciones mencionadas.

Se han desarrollado formas de liberación controlada de esomeprazol en la búsqueda de mejorar la absorción y biodisponibilidad, y evitar así su degradación química temprana debido a que se podría limitar su eficacia. La presentación de esomeprazol NEXIUM-MUPS ${ }^{\circledast}$, desarrollada por AstraZeneca, incluye un sistema que usa micropellets incorporados en la tableta (sistema MUPS) que se liberan con la desintegración de esta, para proteger a este inhibidor de la bomba de protones (IBP). En Colombia, se ha desarrollado una formulación de esomeprazol que utiliza un recubrimiento con un polímero que garantiza la acidorresistencia de las tabletas durante su paso por el medio ácido gástrico y su desintegración al llegar a los $\mathrm{pH}$ menos ácidos $(\mathrm{pH}>4,5)$ en la porción proximal del duodeno.

El objetivo de este estudio fue comparar la respuesta clínica con el uso de dos formulaciones de esomeprazol evaluada en términos de concentraciones de niveles séricos en la primera hora después de la ingesta del medicamento, eficacia por el aumento del $\mathrm{pH}$ gástrico en el seguimiento durante 24 horas, evolución clínica por los cuestionarios de las escalas SODA y QoL-PEI, y la seguridad clínica por los eventos adversos de la terapia con esomeprazol de liberación especial y la terapia con esomeprazol de referencia, ambas de $40 \mathrm{mg} /$ día en pacientes dispépticos con enfermedad orgánica no demostrada.

\section{MATERIALES Y MÉTODOS}

Se realizó un ensayo clínico controlado, aleatorizado a dos brazos, ciego, comparando dos presentaciones de esomeprazol $40 \mathrm{mg} /$ día por 28 días. La población escogida fue los pacientes que asistieron a la consulta de gastroenterología en la Fundación Valle del Lili entre julio de 2016 y marzo de 2017 con síntomas abdominales y digestivos sugestivos de dispepsia, sin estudios previos. Los criterios de inclusión fueron pacientes mayores de 18 años con diagnóstico final de dispepsia no estudiada. Se tomaron como criterios de exclusión la pérdida de peso no explicada, disfagia, anemia, sangrado, ictericia, antecedentes de cirugía gástrica, neoplasias, esofagitis erosiva, embarazo, lactancia, alergia conocida al fármaco del estudio, uso de antiinflamatorios no esteroideos (AINE) o IBP dos semanas antes o fármacos con potenciales interacciones; así mismo, fueron excluidos los casos que tuvieran antecedente de endoscopia de vías digestivas altas (EVDA) con reporte de $\mathrm{pH}$ alcalino, úlcera digestiva o malignidad.

Este estudio se desarrolló en la Fundación Valle del Lili en conformidad con la Declaración de Helsinki 2013, la Conferencia Internacional de Armonización, la Resolución 8430 de 1993, la Resolución 2378 de 2008 de Colombia y la Guía para buenas prácticas clínicas. El estudio fue aprobado por el comité de ética en investigación biomédica y cada participante consintió su ingreso al estudio. Los sujetos no fueron compensados por su participación. Este estudio fue incluido en el registro internacional de Clinical Trials.

En el contexto de un ensayo clínico piloto se buscó incluir al menos a 30 voluntarios en cada grupo para poder probar normalidad en la distribución. Sin embargo, por 
problemas en el reclutamiento solo se lograron incluir a 16 y 17 pacientes en cada grupo.

El estudio se inició realizando un tamizaje de los voluntarios a través de consulta con el gastroenterólogo y la EVDA, en la cual además de la toma de biopsias rutinarias, se midió el pH gástrico con $\mathrm{pH}$ metro Inolab $7110^{\circ}$, tinción con rojo Congo y papel tornasol, para excluir a los pacientes en los que se demostrara causa orgánica o $\mathrm{pH}>4$, sugestivo de hipoclorhidria. Después del tamizaje se realizó la aleatorización a uno de los dos fármacos del estudio, esomeprazol de liberación modificada de Tecnoquímicas (esomeprazol de prueba) o NEXIUM-MUPS ${ }^{\circledR}$ de AstraZeneca (esomeprazol de referencia). Ambos productos están comercializados en Colombia y tienen registro sanitario para la indicación utilizada en este estudio. La secuencia de aleatorización se realizó con el software Randomization ${ }^{\oplus}$, creando bloques de 6 participantes (12). De allí se crearon sobres de seguridad relacionando el código del participante y el grupo al que fue asignado: fármaco $\mathrm{A} \mathrm{o} \mathrm{B}$.

El médico tratante y el equipo de investigación estuvieron ciegos al fármaco aleatorizado durante todo el estudio. Como las presentaciones de los medicamentos eran diferentes, no fue posible asegurar el cegamiento a los sujetos del estudio. El tratamiento suministrado el día del reclutamiento fue una tableta diaria de esomeprazol de $40 \mathrm{mg}$ para 28 días con las indicaciones de tomarla al menos 30 minutos antes del desayuno y hacer el registro en el diario. Luego se programaron dos visitas de seguimiento a las $2 \mathrm{y}$ 4 semanas de estar recibiéndolo para evaluar los desenlaces del estudio (Figura 1).

La evaluación del impacto sobre el $\mathrm{pH}$ gástrico también se llevó a cabo en el seguimiento de las dos semanas después del inicio del tratamiento con esomeprazol, mediante una $\mathrm{pH}$ impedanciometría de 24 horas ambulatoria con catéter Versaflex ${ }^{\oplus} \mathrm{Z}$ dual $\mathrm{pH}$ sensor 8 impedance rings, se almacenó en un equipo digitraper $\mathrm{pH} \mathrm{Z}$ Given ${ }^{\circledast}$ Imagin y posteriormente se descargó y analizó la información con el programa Accuview ${ }^{\circledR} \mathrm{pH}-\mathrm{Z}$ 5.2.

Para correlacionar la medición de la pH-metría, se decidió tomar niveles séricos del medicamento al día 14 de tratamiento como una medida que eventualmente pudiera presentar diferencias en el resultado clínico. Para evaluar la concentración de niveles séricos de esomeprazol se tomó una muestra de sangre venosa $(5 \mathrm{~mL})$ dentro de la primera hora de haber recibido la dosis dos semanas después del inicio de tratamiento, tiempo suficiente para garantizar concentraciones de esomeprazol en la sangre en estado de equilibrio. Se congeló el plasma a $-20^{\circ} \mathrm{C}$ y posteriormente se analizó la muestra con un cromatógrafo líquido UHPLC Lachrom Ultra-VWR, con detector de arreglo de diodos para determinar los niveles séricos de esomeprazol.

Para la evaluación clínica se aplicaron dos escalas el día del reclutamiento y en las dos visitas de seguimiento posteriores ( 2 y 4 semanas después del inicio del tratamiento). La primera escala que se usó fue SODA, la versión validada por el equipo de Benites y colaboradores y, además, el ajuste del puntaje para equilibrar las subescalas sugerido y aplicado por Rabeneck y colaboradores $(10,11)$. Para su análisis esta se divide en las tres subescalas que la componen: intensidad del dolor, síntomas asociados no dolorosos (como eructos, acidez, hinchazón, entre otros) y nivel de conformidad. Las dos primeras subescalas expresan mayor gravedad a mayor puntaje; por el contrario, la subescala de conformidad expresa mayor nivel de bienestar percibido por el paciente a mayor puntaje. La segunda escala, Qol-PEI, se aplicó con la misma metodología y en los mismos tiempos que la escala

\begin{tabular}{|c|c|c|c|c|}
\hline Visita 1 & Visita 2 & $\begin{array}{c}\text { Aleatorización de los } \\
\text { grupos }\end{array}$ & $\begin{array}{c}\text { Visita } 3 \\
\text { Seguimiento a las } 2 \\
\text { semanas }\end{array}$ & $\begin{array}{c}\text { Visita } 4 \\
\text { Seguimiento a las } 4 \\
\text { semanas }\end{array}$ \\
\hline $\begin{array}{l}\text { - Consulta médica } \\
\text { inicial } \\
\text { - Consentimiento } \\
\text { informado }\end{array}$ & $\begin{array}{l}\text { - Endoscopia } \\
\text { digestiva } \\
\text { - Pruebas pH } \\
\text { - pH tornasol } \\
\text { - Rojo congo }\end{array}$ & $\begin{array}{l}\text { - Encuesta calidad } \\
\text { de vida } \\
\text { - SODA } \\
\text { - QOL-pEI } \\
\text { - Inicio con } \\
\text { esomeprazol } \\
\text { - Prueba y de } \\
\text { referencia }\end{array}$ & $\begin{array}{l}\text { - Encuesta calidad } \\
\text { de vida } \\
\text { - SODA } \\
\text { - QOL-pEI } \\
\text { - Consulta médica } \\
\text { - pH-metría } 24 \text { horas } \\
\text { - Niveles séricos de } \\
\text { esomeprazol }\end{array}$ & $\begin{array}{l}\text { - Encuesta calidad } \\
\text { de vida } \\
\text { - SODA } \\
\text { - QOL-pEI }\end{array}$ \\
\hline
\end{tabular}

Figura 1. Distribución de actividades del ensayo clínico 
SODA, pero para su análisis con base en la literatura se tomó un único puntaje global que expresa mayor gravedad de los síntomas a mayor puntaje que, a diferencia del SODA, anexa la incapacidad asociada con el dolor (9).

La evaluación de la seguridad clínica se monitorizó en cada visita a través del análisis del diario que registró el paciente durante el tiempo de tratamiento, en el que asociaron síntomas y eventos adversos.

\section{Análisis estadístico}

Toda la información de los participantes se recolectó en una base de datos con captura electrónica en la plataforma BD Clinic ${ }^{\oplus}$. Se realizó un análisis descriptivo, en el que las variables continuas se expresaron como promedio y desviación estándar (DE), o mediana y rango intercuartílico (RIC); y se compararon con una prueba T de Student o Mann-Whitney, según se cumplió el supuesto de normalidad. Las variables categóricas se presentaron en proporciones y la correlación se realizó con chi cuadrado $\left(\chi_{2}\right)$ o prueba exacta de Fisher, según las observaciones.

Posteriormente, se ejecutó con las escalas clínicas un análisis de varianza (ANOVA) de dos factores (fármacotiempo) con medidas repetidas, que se realizó para establecer las diferencias con un total de 99 repeticiones. Al encontrar diferencias significativas en los tiempos para el efecto principal del ANOVA, se realizó una comparación por pares usando la corrección de Bonferroni para comparaciones múltiples. El análisis se realizó con el paquete estadístico STATA, versión 12.1.

\section{RESULTADOS}

En total se tamizaron 205 pacientes, se reclutaron 55 y se finalizó con una muestra de 33. La principal causa de exclusión fue $\mathrm{pH}$ gástrico sugestivo de hipoclorhidria (Figura 2) (13). Durante el seguimiento hubo pérdida de 2 participantes a quienes no se les pudo realizar $\mathrm{pH}$-metría y se excluyeron del análisis.

Por una parte, la comparación de las características basales sociodemográficas y clínicas se resumen en la Tabla 1, en la que no se encontraron diferencias significativas entre los grupos. En relación con los niveles séricos entre fármacos se encontraron concentraciones para esomeprazol de prueba de $0,67 \mu \mathrm{mol} / \mathrm{L}(0,18-1,61)$ y esomeprazol de referencia de $0,28 \mu \mathrm{mol} / \mathrm{L}(0,16-0,53)$ sin diferencias $(p=0,219)$, al igual que en la comparación sobre la inhibición de la secreción ácida, con un $\mathrm{pH}$ gástrico constante $>4$, que mostró un promedio de tiempo para el esomeprazol de prueba de 19,98 h $(\mathrm{DE} \pm 3,87[83,3 \%])$ y para esomeprazol de referencia de $19,95 \mathrm{~h}(\mathrm{DE} \pm 3,55[83,2 \%]), p=0,986$.

Por otra parte, en el análisis de las escalas de valoración clínica se encontraron diferencias significativas para ambas escalas a las 4 semanas de tratamiento, y se observa en la sub-

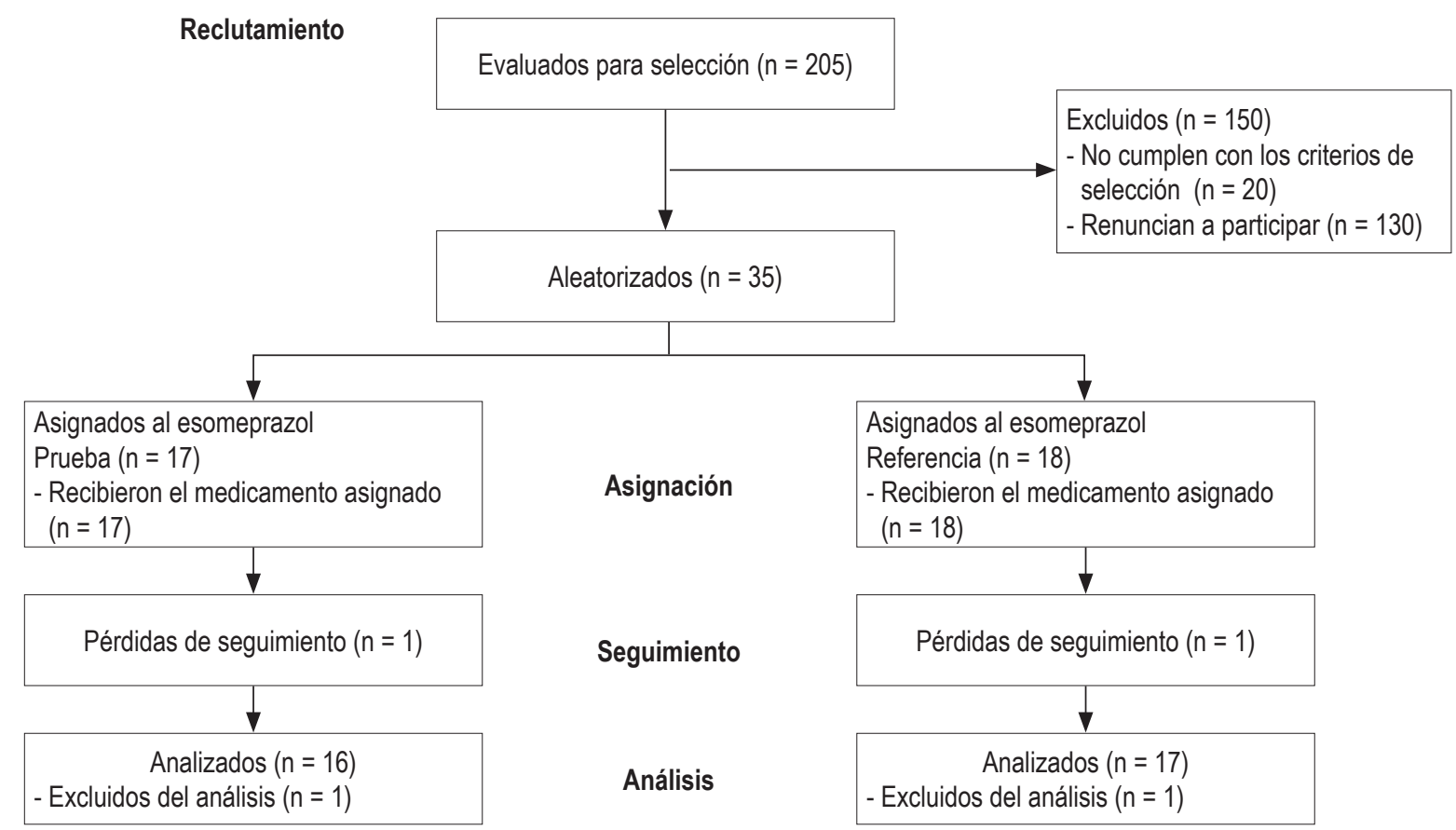

Figura 2. Diagrama de flujo de participantes desde el reclutamiento que inició el mes de Julio del 2016 hasta Abril del 2017 en el ensayo clínico. 
Tabla 1. Comparación de las características basales sociodemográficas y clínicas entre ambos grupos: esomeprazol de prueba $(\mathrm{n}=16)$ y esomeprazol de referencia $(\mathrm{n}=17)$

\begin{tabular}{|c|c|c|c|c|}
\hline Variable & General & Esomeprazol prueba & Esomeprazol referencia & $p$ \\
\hline Sexo & $n=33$ & $n=16$ & $\mathrm{n}=17$ & \multirow{3}{*}{1} \\
\hline Masculino, n (\%) & $8(24,2)$ & $4(25)$ & $4(23,5)$ & \\
\hline Femenino, n (\%) & $25(75,8)$ & $12(75)$ & $13(76,5)$ & \\
\hline Edad $^{*}$ & $38,2 \pm 12,7$ & $37,6 \pm 11,4$ & $38,8 \pm 14,2$ & 0,7919 \\
\hline pH-metría banco** & $1,81(1,61-1,94)$ & $1,7(1,5-2,04)$ & $1,85(1,65-1,9)$ & 0,6266 \\
\hline pH-metría tornasol* & $1,9 \pm 0,68$ & $1,8 \pm 0,72$ & $1,9 \pm 0,66$ & 0,7844 \\
\hline pH-metría rojo congo, $\mathrm{pH}<4$ & $33(100)$ & $16(100)$ & $17(100)$ & - \\
\hline Presencia de H. pylori & $n=33$ & $n=16$ & $\mathrm{n}=17$ & \multirow{3}{*}{0,071} \\
\hline Sí, n (\%) & $22(66,7)$ & $8(50)$ & $14(82,4)$ & \\
\hline No, $\mathrm{n}(\%)$ & $11(33,3)$ & $8(50)$ & $3(17,6)$ & \\
\hline Distribución de H. pylori en el antro $(n=22)$ & $n=22$ & $n=8$ & $n=14$ & \multirow{4}{*}{0,831} \\
\hline Abundante, $\mathrm{n}(\%)$ & $13(39,4)$ & $4(25)$ & $9(52,9)$ & \\
\hline Moderada, n (\%) & $2(6,1)$ & $1(6,25)$ & $1(5,9)$ & \\
\hline Escasa, n (\%) & $7(21,2)$ & $3(18,75)$ & $4(23,5)$ & \\
\hline Distribución de H. pylori en el cuerpo $(n=22)$ & $\mathrm{n}=22$ & $n=8$ & $n=14$ & \multirow{5}{*}{0,858} \\
\hline Abundante, $\mathrm{n}(\%)$ & $9(27,3)$ & $3(18,75)$ & $6(35,3)$ & \\
\hline Moderada, n (\%) & $4(12,1)$ & $1(6,25)$ & $3(17,6)$ & \\
\hline Escasa, $n(\%)$ & $6(18,2)$ & $3(18,75)$ & $3(17,6)$ & \\
\hline Ausente, $\mathrm{n}(\%)$ & $3(9,1)$ & $1(6,25)$ & $2(11,8)$ & \\
\hline Diagnóstico de la biopsia del antro & $n=33$ & $n=16$ & $\mathrm{n}=17$ & \multirow{6}{*}{0,794} \\
\hline Gastritis crónica superficial no atrófica & $28(84,8)$ & $13(81,25)$ & $15(88,2)$ & \\
\hline Gastritis crónica grave sin atrofia & $2(6,1)$ & $1(6,25)$ & $1(5,9)$ & \\
\hline Gastritis crónica con atrofia & $3(9,1)$ & $2(12,5)$ & $1(5,9)$ & \\
\hline Diagnóstico de la biopsia del cuerpo & $n=33$ & $n=16$ & $\mathrm{n}=17$ & \\
\hline Gastritis crónica superficial no atrófica & $31(93,9)$ & $15(93,75)$ & $16(94,1)$ & \\
\hline Gastritis crónica grave sin atrofia & $1(3)$ & $0(0)$ & $1(5,9)$ & \multirow[t]{2}{*}{1} \\
\hline Gastritis crónica con atrofia & $1(3)$ & $1(6,25)$ & $0(0)$ & \\
\hline Porcentaje de tiempo $\mathrm{pH}>4(\mathrm{n}=33)$ & $88,2(71,2-95,6)$ & $88,3(71,3-97,05)$ & $87,3(75,7-91,7)$ & 0,4594 \\
\hline \multicolumn{5}{|l|}{ Eventos adversos, $n=33(\%)$} \\
\hline T1, 2 semanas & $5(15,2)$ & $1(6,3)$ & $4(23,5)$ & 0,335 \\
\hline T2, 4 semanas & $2(6,1)$ & $1(6,3)$ & $1(5,9)$ & 1 \\
\hline
\end{tabular}

${ }^{*}$ Promedio \pm DE. ${ }^{* *}$ Mediana (RIC).

escala de intensidad del dolor la disminución de los puntajes más altos para el esomeprazol de prueba respecto al de referencia, comportamiento similar al observado en la subescala de nivel de conformidad, en la que la mejoría es más notoria en el producto de prueba (Tabla 2). Estas tendencias se repitieron nuevamente en el análisis ANOVA de dos factores en los que hubo diferencias en la variable tiempo, pero solamente con interacción en la subescala dolor; en la subescala de conformidad se encontraron diferencias entre los tratamientos $(p=0,012)$ (Tabla 3). En el ajuste de Bonferroni se encontró que de manera independiente para cada Esomeprazol, hay diferencias significativas $(p<0,05)$ entre el tiempo de inicio y final del tratamiento a las 4 semanas, tanto en cada una de las subescalas de SODA como la escala QoLPEI; sin embargo, en la subescala en SODA dolor no hubo diferencias en este tiempo $(p=0,018)$. 
Tabla 2. Comparación de los fármacos entre tiempos independientes, tomando como línea de base el tiempo 0 al momento del reclutamiento y las visitas de seguimiento a las 2 semanas como tiempo 1 y a las 4 semanas como tiempo 2.

\begin{tabular}{|c|c|c|c|c|}
\hline ESCALA SODA $^{* *} Y$ QOL-PE| $\left.\right|^{* * *}$ & $\begin{array}{c}\text { General } \\
n=33\end{array}$ & $\begin{array}{c}\text { Esomeprazol TQ } \\
n=16\end{array}$ & $\begin{array}{c}\text { Esomeprazol AZ } \\
n=17\end{array}$ & $p$ \\
\hline \multicolumn{5}{|l|}{ SODA Intensidad del dolor* (Rango 2-47) } \\
\hline Tiempo 0 (sin tratamiento) & $25,8 \pm 5,5$ & $24,4 \pm 6,4$ & $27,2 \pm 4,4$ & 0,1603 \\
\hline Tiempo 1 (2 semanas de tratamiento) & $19,5 \pm 6,2$ & $19,75 \pm 7,15$ & $19,3 \pm 5,4$ & 0,8376 \\
\hline Tiempo 2 (4 semanas de tratamiento) & $16,6 \pm 9,8$ & $12,18 \pm 9,3$ & $20,8 \pm 8,5$ & 0,0087 \\
\hline \multicolumn{5}{|l|}{ SODA Intensidad síntomas asociados* (Rango 7-35) } \\
\hline Tiempo 0 (Sin tratamiento) & $17,8 \pm 2,7$ & $17,6 \pm 1,8$ & $18 \pm 3,3$ & 0,6911 \\
\hline Tiempo 1 (2 semanas de tratamiento) & $14,1 \pm 2,9$ & $13,9 \pm 2,9$ & $14,3 \pm 2,9$ & 0,7273 \\
\hline Tiempo 2 ( 4 semanas de tratamiento) & $13,9 \pm 4,04$ & $12,7 \pm 3,9$ & $15 \pm 3,9$ & 0,1118 \\
\hline \multicolumn{5}{|l|}{ SODA Nivel de conformidad* (Rango 2-23) } \\
\hline Tiempo 0 (Sin tratamiento) & $7,4 \pm 3,2$ & $8,2 \pm 3,1$ & $6,7 \pm 3,3$ & 0,2113 \\
\hline Tiempo 1 ( 2 semanas de tratamiento) & $12,1 \pm 5,1$ & $12,7 \pm 5,6$ & $11,5 \pm 4,7$ & 0,5046 \\
\hline Tiempo 2 (4 semanas de tratamiento) & $14,5 \pm 5,5$ & $17,2 \pm 5,2$ & $11,9 \pm 4,4$ & 0,0035 \\
\hline \multicolumn{5}{|l|}{ QoL-PEI* (rango 12-120) } \\
\hline Tiempo 0 (Sin tratamiento) & $65,5 \pm 17,3$ & $64 \pm 17,3$ & $66,8 \pm 17,69$ & 0,6401 \\
\hline Tiempo 1 (2 semanas de tratamiento) & $38,6 \pm 13,4$ & $37,5 \pm 13,54$ & $39,58 \pm 13,58$ & 0,6616 \\
\hline Tiempo 2 ( 4 semanas de tratamiento) & $39,7 \pm 18,5$ & $32,68 \pm 14,2$ & $46,29 \pm 19,32$ & 0,029 \\
\hline
\end{tabular}

${ }^{*}$ Promedio \pm DE Two-sample t test. ${ }^{* *}$ Severity of dyspepsia assessment. ${ }^{* * *}$ Cuestionario de calidad de vida asociado con problemas intestinales.

Tabla 3. Comparación entre ambos fármacos mediante ANOVA de medidas repetidas de dos factores, tomando como línea de base el tiempo 0 al momento del reclutamiento y las visitas de seguimiento a las 2 semanas como tiempo 1 y a las 4 semanas como tiempo 2

\begin{tabular}{|c|c|c|c|c|c|c|c|}
\hline \multicolumn{8}{|c|}{ SODA $^{* *}$ Y QOL-PE| ${ }^{\star * *}$ ANOVA MR } \\
\hline Escala & Fármaco & Tiempo 0 & Tiempo 1 & Tiempo 2 & trt & time & trt\#time \\
\hline \multirow[t]{2}{*}{ SODA DOLOR } & $\mathrm{TQ}^{*}$ & $24,4 \pm 6,4$ & $19,75 \pm 7,15$ & $12,18 \pm 9,3$ & \multirow{2}{*}{0,0623} & \multirow{2}{*}{0} & \multirow{2}{*}{0,0048} \\
\hline & $A Z^{*}$ & $27,2 \pm 4,4$ & $19,3 \pm 5,4$ & $20,8 \pm 8,5$ & & & \\
\hline \multirow{2}{*}{$\begin{array}{l}\text { SODA síntomas } \\
\text { asociados }\end{array}$} & $\mathrm{TQ}^{*}$ & $17,6 \pm 1,8$ & $13,9 \pm 2,9$ & $12,7 \pm 3,9$ & \multirow{2}{*}{0,2215} & \multirow{2}{*}{0} & \multirow{2}{*}{0,2973} \\
\hline & $A Z^{*}$ & $18 \pm 3,3$ & $14,3 \pm 2,9$ & $15 \pm 3,9$ & & & \\
\hline \multirow[t]{2}{*}{ SODA conformidad } & $T Q^{*}$ & $8,2 \pm 3,1$ & $12,7 \pm 5,6$ & $17,2 \pm 5,2$ & \multirow{2}{*}{0,012} & \multirow{2}{*}{0} & \multirow{2}{*}{0,0968} \\
\hline & $A Z^{*}$ & $6,7 \pm 3,3$ & $11,5 \pm 4,7$ & $11,9 \pm 4,4$ & & & \\
\hline \multirow[t]{2}{*}{ QoL-PEI } & $T Q^{*}$ & $64 \pm 17,3$ & $37,5 \pm 13,54$ & $32,68 \pm 14,2$ & \multirow{2}{*}{0,1572} & \multirow{2}{*}{0} & \multirow{2}{*}{0,1357} \\
\hline & $A Z^{*}$ & $66,8 \pm 17,69$ & $39,58 \pm 13,58$ & $46,29 \pm 19,32$ & & & \\
\hline
\end{tabular}

${ }^{*}$ Promedio \pm DE. ${ }^{* *}$ Severity of dyspepsia assessment. ${ }^{* * *}$ Cuestionario de calidad de vida asociado a problemas intestinales.

La proporción de pacientes que experimentaron eventos adversos durante el estudio fue similar entre ambos grupos con una frecuencia a las 4 semanas para esomeprazol TQ de $6,3 \%$ y para esomeprazol AZ de 5,9 \% $(p=0,999)$, todos los eventos fueron no serios con resolución antes de finalizar el estudio, asociados con el uso de los fármacos y contemplados en las fichas técnicas como: náuseas, resequedad en la boca, eructos y flatulencias.

\section{DISCUSIÓN}

Los resultados de las concentraciones séricas medidas en nuestros pacientes fueron muy variables, característica similar a lo referido en la literatura. Se plantea que esta diferencia puede deberse al diseño de sus presentaciones farmacéuticas de liberación especial, tiempo de la toma, condición fisiológica al momento del consumo del fármaco 
y, de otra parte, a la capacidad del individuo de metabolizar el IBP según los polimorfismos en sus enzimas, especialmente la CYP2C19 (6, 14-20).

En referencia a la efectividad sobre el $\mathrm{pH}$ gástrico, ambos fármacos evidenciaron una alta inhibición de la producción de ácido (83\%), valor que estaría en los límites superiores en relación con lo referido en estudios similares (50\% y $85 \%)$; y cuyo valor mínimo de inhibición en nuestro estudio fue del $50 \%$ y se considera similar al establecido para tener un impacto clínico $(7,8,14,15)$. No hubo diferencias en los niveles séricos del medicamento al día 14, lo cual pudiere explicar en parte este hallazgo; sin embargo, los niveles séricos no se correlacionan necesariamente con los niveles intracelulares.

En relación con la evolución clínica se evidenciaron descensos significativos en los puntajes de la escala SODA. En el presente estudio, al analizar su comportamiento se encontró en la subescala de dolor caídas de 20,8 y 12,8 puntos en esomeprazol TQ y esomeprazol AZ respectivamente, valores un poco más altos que los reportados por Benites y colaboradores, que describieron descensos de 7 puntos en esta subescala; no obstante, en la subescala de síntomas no asociados con el dolor se tuvieron descensos bajos de alrededor de 2,5 puntos, similares a los encontrados en ese estudio. Paralelamente, se encontró un comportamiento similar a los hallazgos de Benites y colaboradores $y$ en otro estudio semejante de Rabeneck y colaboradores, cuyo mayor impacto se dio en la subescala de dolor. Concluyeron que este resultado es posible al haber realizado un control efectivo de ácido gástrico, que es la principal causa de sensación dolorosa, y al estar las preguntas sobre conformidad asociadas con el control del dolor. En la evaluación de los cambios en la subescala de conformidad se observó que el esomeprazol de prueba tuvo un puntaje final más alto de mejoría clínica respecto al de referencia, con un puntaje promedio de 9 puntos de mejoría en el primero y 5,2 puntos en el segundo $(p=0,0035)$; dichos aumentos también fueron más altos que los reportados por Benites y colaboradores, que fueron de 2,5 puntos en su población para este aspecto $(10,11)$.

La frecuencia general de eventos adversos a las 4 semanas en este estudio fue de 6,3\% para esomeprazol de prueba y de 5,9\% para esomeprazol de referencia; frecuencias bajas comparadas con lo encontrado por Shin y colaboradores, que reportaron el $40 \%$ en una muestra de 36 pacientes, pero coincide con ellos en que fueron todos eventos adversos no serios, efecto que se ha descrito además en diversas publicaciones en las que se ha encontrado que estos fármacos cuentan con un adecuado perfil de seguridad farmacológico en usos no prolongados (14-21).

$\mathrm{Al}$ analizar las limitaciones del presente estudio, se encuentra que el proceso de reclutamiento evidenció que, a pesar de que la dispepsia es una patología con alta prevalencia, su diagnóstico y tamizaje para la selección de pacientes es complejo buscando excluir organicidad, por lo que se usó la consulta clínica y endoscópica, pero no otros medios diagnósticos como la ecografía y laboratorios que pudieran descartar otras causas.

Por otra parte, al haber sido desarrollado como un estudio piloto, al momento del cierre se encontró una potencia estadística baja; si se quisiera tomar una muestra representativa para esta patología con prevalencia del $10 \%$ al $30 \%$ de la población general y potencia del 80 $\%$, se requerirían al menos 1500 pacientes $(22,23)$. El tiempo de seguimiento y efectividad en el estudio fue de 4 semanas; por tanto, no se pueden extrapolar los datos a situaciones clínicas para uso de estos medicamentos de manera más prolongada. Además, no cegar a los pacientes para la toma del medicamento pudo haber introducido sesgo de selección a nuestro estudio, lo que pudo tener algún efecto en los resultados encontrados.

Se concluyó al realizar la comparación entre fármacos que ambos mostraban comportamientos muy similares respecto a los desenlaces de interés, aumento del $\mathrm{pH}$ y el cuestionario de síntomas. Respecto a la evolución de las escalas de valoración clínica, no se observaron diferencias importantes entre los tratamientos, pero sí se puede apreciar que en el grupo de pacientes con el esomeprazol de prueba, el efecto se sostiene mejor a través del tiempo, en comparación con el esomeprazol de referencia, diferencia con significancia estadística; valdría la pena considerar si esto pudo haber sido secundario a un mayor número de casos positivos para $H$. pylori en el grupo de esomeprazol de referencia, aunque este factor no representó una diferencia estadísticamente significativa $(p=0,071)$. Respecto a la seguridad, ambos mostraron ser IBP de bajo riesgo para un consumo en este período y en la población estudiada.

\section{Agradecimientos}

A la unidad de endoscopia por la colaboración para la ejecución del estudio. A todo el equipo parte del centro de investigaciones clínicas por sus aportes. Al Laboratorio de instrumentación química de la Universidad ICESI por el análisis de las concentraciones séricas.

\section{Conflicto de interés}

No se declaran conflictos de interés.

\section{Fuentes de financiación}

\section{Tecnoquímicas.}




\section{REFERENCIAS}

1. Talley NJ, Vakil N. Guidelines for the management of dyspepsia. Am J Gastroenterol. 2005;100(10):2324-37. doi: 10.1111/j.1572-0241.2005.00225.x.

2. Stanghellini V, Chan FK, Hasler WL, Malagelada JR, Suzuki $\mathrm{H}$, Tack J, et al. Gastroduodenal disorders. Gastroenterology. 2016;150(6):1380-92. doi: 10.1053/j.gastro.2016.02.011.

3. Talley NJ. Functional dyspepsia: new insights into pathogenesis and therapy. Korean J Intern Med. 2016;31(3):44456. doi: 10.3904/kjim.2016.091.

4. Bravo LE, Cortés A, Carrascal E, Jaramillo R, García LS, Bravo PE, et al. Helicobacter pylori: patología y prevalencia en biopsias gástricas en Colombia. Colomb Med. 2003;34(3):124-31.

5. Otero W, Zuleta MG, Otero L. Enfoque del paciente con dispepsia y dispepsia funcional: actualización. Rev Col Gastroenterol. 2014;29(2):132-8.

6. Shin JM, Kim N. Pharmacokinetics and pharmacodynamics of the proton pump inhibitors. J Neurogastroenterol Motil. 2013;19(1):25-35. doi: 10.5056/jnm.2013.19.1.25.

7. Hatlebakk JG. Review article: gastric acidity - comparison of esomeprazole with other proton pump inhibitors. Alimentary Pharmacology \& Therapeutics. 2003;17:10-5. doi: 10.1046/j.1365-2036.17.s1.3.x.

8. Kirchheiner J, Glatt S, Fuhr U, Klotz U, Meineke I, Seufferlein $\mathrm{T}$, et al. Relative potency of proton-pump inhibitors-comparison of effects on intragastric $\mathrm{pH}$. Eur J Clin Pharmacol. 2009;65(1):19-31. doi: 10.1007/s00228-008-0576-5.

9. Ruiz M, Villasante F, León F, González-Lara V, González C, Crespo $\mathrm{M}$, et al. Cuestionario sobre calidad de vida asociada a dispepsia. Adaptación española y validación del cuestionario Dyspepsia Related Health Scale. Medicina Clínica. 2001;117(15):567-73. doi: 10.1016/S00257753(01)72182-3.

10. Benites Goñi $H$, Cabrera Cabrejos S, Chungui Bravo J, Prochazka Zarate R, Bernabe Ortiz A, De los Ríos Senmache $\mathrm{R}$, et al. Modificación y validación del instrumento SODA (severity of dyspepsia assessment) adaptada al Perú para evaluar la evolución de la severidad de los síntomas en pacientes con dispepsia. Rev Gastroenterol Perú. 2013;33(1):9-27.

11. Rabeneck L, Cook KF, Wristers K, Souchek J, Menke T, Wray NP. SODA (severity of dyspepsia assessment): a new effective outcome measure for dyspepsia-related health. Journal of clinical epidemiology. 2001;54(8):755-65. doi: 10.1016/S0895-4356(00)00365-6.

12. Randomization.com. [internet] 2007 [acceso el 15 de marzo de 2017]. Disponible en: http://www.randomization.com/2007.

13. Moher D, Hopewell S, Schulz KF, Montori V, Gøtzsche PC, Devereaux PJ, et al. CONSORT 2010 Explanation and Elaboration: updated guidelines for reporting parallel group randomised trials. BMJ. 2010;340:c869. doi: 10.1136/bmj. c869.

14. Ullah MA, Shams Ud D, Maruf AA, Azad MAK, Shohag $\mathrm{MH}$, Sultana R, et al. Relative bioavailability and pharmacokinetic properties of two different enteric formulations of esomeprazole in healthy bangladeshi male volunteers: An open-label, single-dose, randomized-sequence, two-way crossover study. Clinical Therapeutics. 2010;32(7):141926. doi: 10.1016/j.clinthera.2010.07.007.

15. Shin JS, Lee JY, Cho KH, Park HL, Kukulka M, Wu JT, et al. The pharmacokinetics, pharmacodynamics and safety of oral doses of ilaprazole 10,20 and $40 \mathrm{mg}$ and esomeprazole $40 \mathrm{mg}$ in healthy subjects: a randomised, open-label crossover study. Alimentary Pharmacology \& Therapeutics. 2014;40(5):548-61. doi: 10.1111/apt.12860.

16. Furuta T, Ohashi K, Kosuge K, Zhao XJ, Takashima M, Kimura M, et al. CYP2C19 genotype status and effect of omeprazole on intragastric $\mathrm{pH}$ in humans. Clinical Pharmacology \& Therapeutics. 1999;65(5):552-61. doi: 10.1016/S0009-9236(99)70075-5.

17. Hunfeld NG, Touw DJ, Mathot RA, van Schaik RH, Kuipers EJ. A comparison of the acid-inhibitory effects of esomeprazole and rabeprazole in relation to pharmacokinetics and CYP2C19 polymorphism. Alimentary Pharmacology \& Therapeutics. 2012;35(7):810-8. doi: $10.1111 /$ j.13652036.2012.05014.x.

18. Klotz U. Impact of CYP2C19 polymorphisms on the clinical action of proton pump inhibitors (PPIs). Eur J Clin Pharmacol. 2009;65(1):1-2. doi: 10.1007/s00228-0080571-x.

19. Dean L. Esomeprazole Therapy and CYP2C19 Genotype. 2012 [updated 2016 Mar 8]. In: Pratt V, McLeod H, Rubinstein W, Dean L, Kattman B, Malheiro A, editors. Medical Genetics Summaries [Internet]. Bethesda (MD): National Center for Biotechnology Information (US); 2012-. Available from http://www.ncbi.nlm.nih.gov/ books/NBK100896/.

20. Lou H-Y, Chang C-C, Sheu M-T, Chen Y-C, Ho H-O. Optimal dose regimens of esomeprazole for gastric acid suppression with minimal influence of the CYP2C19 polymorphism. Eur J Clin Pharmacol. 2008;65(1):55-64. doi: 10.1007/s00228-008-0552-0.

21. MenéndezJLT. Farmacología de esomeprazol. Emergencias: Revista de la Sociedad Española de Medicina de Urgencias y Emergencias. 2005;17(4):1059-66.

22. Mahadeva S, Goh KL. Epidemiology of functional dyspepsia: a global perspective. World J Gastroenterol. 2006;12(17):2661-6. doi: 10.3748/wjg.v12.i17.2661.

23. Talley NJ, Vakil NB, Moayyedi P. American gastroenterological association technical review on the evaluation of dyspepsia. Gastroenterology. 2005;129(5):1756-80. doi: 10.1053/j.gastro.2005.09.020. 